\title{
How to see waves under the Earth surface (the BC-method for geophysicists)
}

\author{
M.I.Belishev*
}

\begin{abstract}
The BC-method is an approach to inverse problems based on their relations to the boundary control theory. The paper provides a simple and physically transparent description of the method in the case of dynamical inverse data given at a portion of the boundary.
\end{abstract}

\section{Introduction}

The BC-method is an approach to inverse problems based on their relations to the boundary control theory. The goal of this paper is to provide a maximally simple and physically transparent description of the approach in the case of dynamical inverse data given at a portion of the boundary. It is the variant of the BC-method, which is most promising for applications in geophysics and acoustics.

A specific feature of the BC-method is that it recovers (visualizes) the waves, whereas recovering the parameters, in a sense, turns out to be a "free addition". The method is time-optimal: the longer is the time of measurements at the surface, the greater is the depth of recovering the waves and parameters.

*The original version is published in Ill-Posed and Inverse Problems. S.I.Kabanikhin and V.G.Romanov (Eds). VSP, 2002, 55-72. 


\section{Geometry of rays}

\section{$1.1 \quad$ c-metric}

We denote by $x=\left(x^{1}, x^{2}, x^{3}\right)$ the points of the space $\mathbb{R}^{3}$.

Let $\Omega \subset \mathbb{R}^{3}$ be a domain (possibly, unbounded) with a smooth boundary $\Gamma$, and let $c=c(x)$ be the speed of waves propagating in $\Omega$. We assume that $0<c_{*} \leqslant c(x) \leqslant c^{*}<\infty$ holds for some constants $c_{*}, c^{*}$.

The speed determines the c-metric with a distance

$$
\tau(x, y):=\inf \int_{x}^{y} \frac{|\mathrm{d} s|}{c(s)}
$$

where the infimum is taken over the set of smooth curves connecting $x$ with $y$; thus $\tau(x, y)$ is the travel time needed for a wave initiated at $x$ to reach $y$. The geodesics of the c-metric are the curves realizing the infimum in (1.1). If $c(x) \equiv 1$, the c-metric is Euclidean: $\tau(x, y)=|x-y|$ and the corresponding geodesics are straight lines.

Let $\sigma \subset \Gamma$ be a portion (open subset) of the boundary. Introduce an eikonal

$$
\tau(x):=\min _{y \in \sigma} \tau(x, y)
$$

and its level surfaces (fronts)

$$
\Gamma^{\xi}:=\{x \in \Omega \mid \tau(x)=\xi\}, \quad \xi \geq 0 .
$$

In dynamics, $\Gamma^{\xi}$ is a forward front (at the moment $t=\xi$ ) of the wave initiated at $\sigma$ and moving into $\Omega$, and $\tau(x)$ is the time needed for the wave to reach the point $x$. Let

$$
\Omega^{\xi}:=\{x \in \Omega \mid \tau(x)<\xi\}
$$

be the subdomain filled with waves at $t=\xi$. This subdomain is bounded by $\Gamma^{\xi}$ and $\Gamma$.

\subsection{Ray coordinates}

Fix a point $\gamma \in \sigma$; let $r_{\gamma}$ be the geodesic of the c-metric starting from $\gamma$ orthogonally to $\sigma$. We shall denote by $r_{\gamma}[0, \xi]$ a segment of $r_{\gamma}$ of the clength $\xi$; let $x(\gamma, \xi)$ be a second endpoint of this segment. 
Fix $T>0$; the rays starting from $\sigma$ cover the subdomain

$$
B^{T}:=\bigcup_{\gamma \in \sigma} \bigcup_{0 \leq \xi \leq T} x(\gamma, \xi)
$$

which we call a tube, $\sigma$ being its bottom. The tube can be represented as a collection of the cross-sections

$$
\sigma^{\xi}:=B^{T} \cap \Gamma^{\xi}=\bigcup_{\gamma \in \sigma} x(\gamma, \xi)
$$

each $\sigma^{\xi}$ being a part of the front $\Gamma^{\xi}$ lighted with rays. Notice that $\sigma^{0}$ coincides with $\sigma$.

As is known, if $T$ is not too large, then the families of rays $r_{\gamma}$ and fronts $\sigma^{\xi}$ are regular. This enables one to introduce a special coordinate system in $B^{T}$. The pair $(\gamma, \xi)$ is said to be the ray coordinates of a point $x \in B^{T}$ if $x$ lies at the ray $r_{\gamma}$ and the front $\sigma^{\xi}$ (i.e., if $x=x(\gamma, \xi)$ ). Notice that $x(\gamma, 0)=\gamma$.

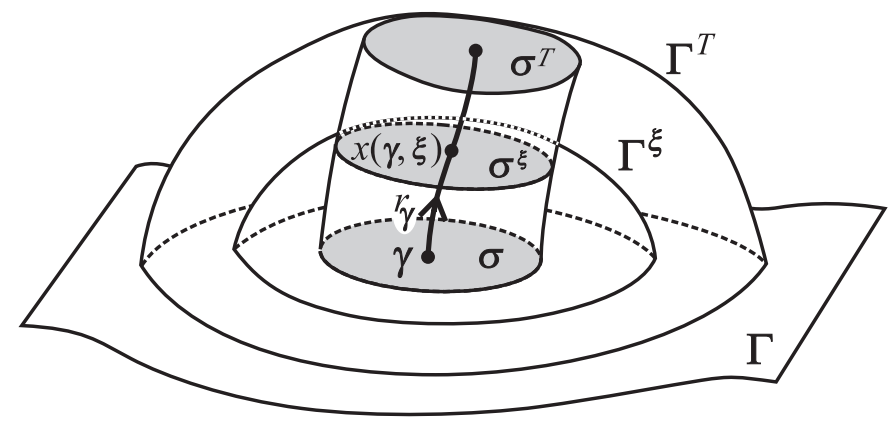

Fig. 1. Ray coordinates

Actually, since $\gamma$ is a point of the boundary, speaking about coordinates we should provide $\gamma$ with local coordinates $\gamma^{1}, \gamma^{2}$ on $\sigma$ but such details are not substantial for what follows.

\subsection{Ray divergence}

An important characteristic of the ray coordinates is a ray divergence, which plays the role of an amplitude factor in the Geometric Optics formulas.

Fix $\gamma \in \sigma$ and denote by $\sigma_{\varepsilon}(\gamma)$ the intersection of $\sigma$ with the ball of small radius $\varepsilon$ centered at $\gamma$. Consider the tube $B_{\varepsilon}^{T}$ formed by rays starting 
from $\sigma_{\varepsilon}(\gamma)$; let $\sigma_{\varepsilon}^{\xi}(\gamma):=B_{\varepsilon}^{T} \cap \Gamma^{\xi}$, and let $|\sigma|$ be an area of $\sigma$. A function of the ray coordinates

$$
J(\gamma, \xi):=\lim _{\varepsilon \rightarrow 0} \frac{\left|\sigma_{\varepsilon}^{\xi}(\gamma)\right|}{\left|\sigma_{\varepsilon}(\gamma)\right|}
$$

is called the ray divergence at the point $x(\gamma, \xi)$ (see, e.g., [1]).

\section{Propagation of jumps}

\subsection{Dynamical system with boundary control}

We denote by $Q^{T}:=\Omega \times(0, T)$ a space-time cylinder; let $\Sigma^{T}:=\Gamma \times[0, T]$ be its lateral surface. The part $\Sigma_{\sigma}^{T}:=\sigma \times[0, T]$ of the lateral surface is referred to as the screen; the meaning of this term will be clarified later.

Consider the boundary initial-value problem

$$
\begin{aligned}
& u_{t t}-c^{2}[\Delta u-q u]=0 \quad \text { in } Q^{T}, \\
& \left.u\right|_{t=0}=\left.u_{t}\right|_{t=0}=0 \quad \text { in } \Omega, \\
& u=f \quad \text { on } \Sigma^{T},
\end{aligned}
$$

where $q=q(x)$ is a potential in $\Omega$ and $f=f(\gamma, t)$ is a boundary control. Let $u=u^{f}(x, t)$ be a solution. This solution describes a wave produced by the control $f$ and moving into the domain from the boundary.

In what follows we deal only with controls supported at the screen: supp $f \subset \Sigma_{\sigma}^{T}$ (i.e., $f(\gamma, t)=0$ for $\gamma \in \Gamma \backslash \sigma$ and for any $t$ ). At the moment $t=\xi$ the forward front of the corresponding wave $u^{f}$ coincides with the surface $\Gamma^{\xi}$ and the wave is supported in the subdomain $\Omega^{\xi}$ :

$$
\operatorname{supp} u^{f}(\cdot, \xi) \subset \Omega^{\xi},
$$

i.e., $u^{f}(x, \xi)=0$ in $\Omega \backslash \Omega^{\xi}$. At the final moment the wave captures the subdomain $\Omega^{T}$.

If the control is switched on with a delay $T-\xi$ (i.e., $\left.f\right|_{0 \leqslant t \leqslant T-\xi}=0$, so that $f$ acts $\xi$ units of time), then at the final moment $t=T$ the wave $u^{f}(\cdot, T)$ is supported in $\Omega^{\xi}$. 


\subsection{Geometric Optics formula}

Take a smooth control $f=f(\gamma, t)$ and fix an intermediate moment $t=T-\xi$, $0<\xi<T$. Let

$$
f_{\xi}(\gamma, t):= \begin{cases}0, & 0 \leqslant t<T-\xi \\ f(\gamma, t), & T-\xi \leqslant t \leqslant T\end{cases}
$$

be the truncated control. An important fact is that the truncation violates the smoothness and leads to appearance of a jump at the moment $t=T-\xi$ :

$$
f_{\xi}(\gamma, T-\xi+0)=f(\gamma, T-\xi)
$$

so that the amplitude of the jump at the point $(\gamma, T-\xi) \in \Sigma_{\sigma}^{T}$ is equal to the value of the original control $f$ at this point.

The well-known fact is that a discontinuous control produces a discontinuous wave with a jump propagating along rays with speed $c(x)$. In our case the wave $u^{f_{\xi}}$ produced by the truncated control enters into the domain with the initial jump

$$
u^{f_{\xi}}(x(\gamma, 0), T-\xi+0) \stackrel{(2.4)}{=} f_{\xi}(\gamma, T-\xi+0) \stackrel{(2.6)}{=} f(\gamma, T-\xi) .
$$

Then this jump propagates along the ray $r_{\gamma}$ and at the final moment $t=T$ arrives at the point $x(\gamma, \xi)$ (see Fig. 2). Its final amplitude $u^{f_{\xi}}(x(\gamma, \xi-0), T)$ is connected with the initial one through the well-known Geometric Optics law:

$$
\frac{u^{f_{\xi}}(x(\gamma, \xi-0), T)}{u^{f_{\xi}}(x(\gamma, 0), T-\xi+0)}=\sqrt{\frac{c(x(\gamma, \xi))}{J(\gamma, \xi)}} / \sqrt{\frac{c(x(\gamma, 0))}{J(\gamma, 0)}}
$$

(see, e.g., [1]), which implies

$$
u^{f}(x(\gamma, \xi-0), T) \stackrel{(2.7)}{=} \sqrt{\frac{c(x(\gamma, \xi)) J(\gamma, 0)}{c(x(\gamma, 0)) J(\gamma, \xi)}} f(\gamma, T-\xi) .
$$

Thus, at the final moment $t=T$, the wave $u^{f_{\xi}}$ occupies the subdomain $\Omega^{\xi}$ and its forward front takes up the position $\Gamma^{\xi}$. At the part $\sigma^{\xi}$ of the front, which is lighted by the rays $r_{\gamma}$, the wave has the jump, whose amplitude can be calculated by $(2.8)$.

Note in addition that the part $\Gamma^{\xi} \backslash \sigma^{\xi}$ of the front may contain singularities of rather complicated structure. Fortunately, this part of the front plays no role in further considerations. 


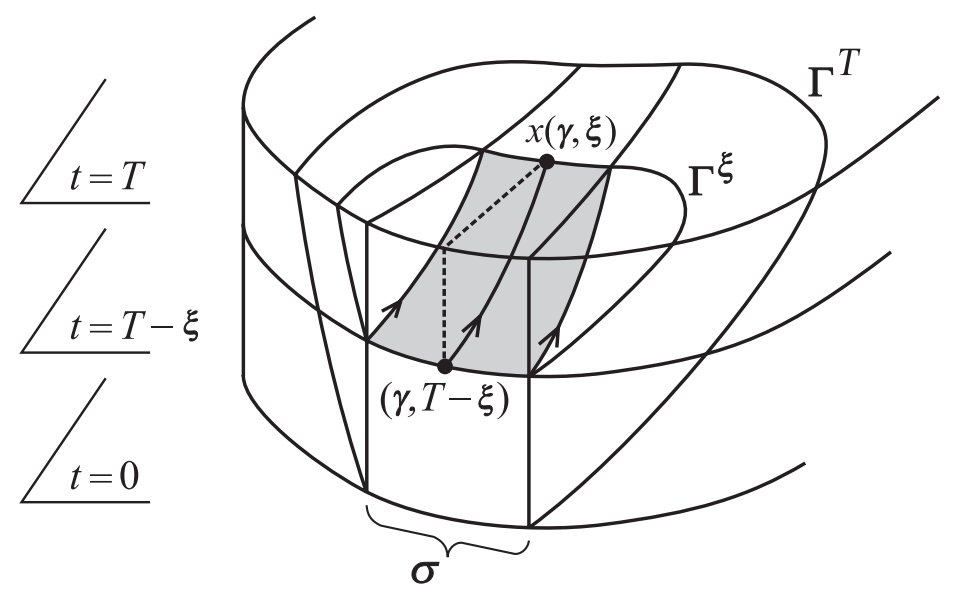

Fig. 2. The jump of $u^{f_{\xi}}$

\subsection{Dual system}

A dynamical system

$$
\begin{aligned}
& v_{t t}-c^{2}[\Delta v-q v]=0 \quad \text { in } Q^{T}, \\
& \left.v\right|_{t=T}=0,\left.\quad v_{t}\right|_{t=T}=y \quad \text { in } \Omega, \\
& v=0 \quad \text { on } \Sigma^{T}
\end{aligned}
$$

is called dual to system (2.2)-(2.4). We denote its solution by $v=v^{y}(x, t)$. Note that the Cauchy data (2.10) are posed at the final moment; this is not essential in view of the well-known time reversibility of equation (2.9).

The solution $v^{y}$ describes a wave, which is produced by a speed perturbation $y=y(x)$ and propagates in the domain with the rigidly fixed boundary $\Gamma$. A function $\partial v^{y}(\gamma, t) / \partial \nu(\nu=\nu(\gamma)$ is an outward normal to $\Gamma)$ is proportional to the force, which appears as a result of interaction between the wave $v^{y}$ and the boundary at the point $\gamma$ at the moment $t$.

Let us derive a relation between the solutions $u^{f}$ and $v^{y}$ of the systems (2.2)-(2.4) and (2.9)-(2.11). It is the relation, which motivates the term "dual": for any control $f$ and perturbation $y$ the relation

$$
\int_{\Omega} \frac{\mathrm{d} x}{c^{2}(x)} u^{f}(x, T) y(x)=\int_{\Sigma^{T}} \mathrm{~d} \Gamma \mathrm{d} t f(\gamma, t) \frac{\partial v^{y}}{\partial \nu}(\gamma, t) .
$$

holds. Indeed, integrating by parts in the equalities

$$
0=\int_{0}^{T} \mathrm{~d} t \int_{\Omega} \frac{\mathrm{d} x}{c^{2}(x)}\left\{u_{t t}^{f}(x, t)-c^{2}(x)\left[\Delta u^{f}(x, t)-q(x) u^{f}(x, t)\right]\right\} v^{y}(x, t)
$$




$$
\begin{aligned}
= & \left.\int_{\Omega} \frac{\mathrm{d} x}{c^{2}(x)}\left[u_{t}^{f}(x, t) v^{y}(x, t)-u^{f}(x, t) v_{t}^{y}(x, t)\right]\right|_{t=0} ^{t=T} \\
& -\int_{0}^{T} \mathrm{~d} t \int_{\Gamma} \mathrm{d} \Gamma\left[\frac{\partial u^{f}}{\partial \nu}(\gamma, t) v^{y}(\gamma, t)-u^{f}(\gamma, t) \frac{\partial v^{y}}{\partial \nu}(\gamma, t)\right] \\
& +\int_{0}^{T} \mathrm{~d} t \int_{\Omega} \frac{\mathrm{d} x}{c^{2}(x)} u^{f}(x, t)\left\{v_{t t}^{y}(x, t)-c^{2}(x)\left[\Delta v^{y}(x, t)-q(x) v^{y}(x, t)\right]\right\}
\end{aligned}
$$

(see $(2.2),(2.3),(2.9)$, and $(2.10))$

$$
=-\int_{\Omega} \frac{\mathrm{d} x}{c^{2}(x)} u^{f}(x, T) y(x)+\int_{0}^{T} \mathrm{~d} t \int_{\Gamma} \mathrm{d} \Gamma f(\gamma, t) \frac{\partial v^{y}}{\partial \nu}(\gamma, t)
$$

we obtain (2.12). To justify these calculations, one needs to begin with taking the control $f$ smooth and vanishing near $t=0$, so that the wave $u^{f}(x, t)$ turns out to be smooth in $\Omega$ and vanishing near its forward front. Due to the latter the surface integrals over the forward front vanish when we integrate by parts. Then the final result (2.12) is extended to a wide class of (possibly discontinuous) controls $f$. Notice also that the integral in the right-hand side of (2.12) is in fact taken over $\Sigma_{\sigma}^{T}$ because the controls are supported on the screen.

In what follows we write (2.12) in a convenient symbolic form. Introduce a control operator $W^{T}$ associated with the system (2.2)-(2.4), which maps controls to a waves:

$$
W^{T} f:=u^{f}(\cdot, T) \quad \text { in } \Omega .
$$

It can be considered as an operator, which creates the waves. Introduce also the observation operator $O^{T}$ associated with the dual system, which maps perturbation $y$ to the force produced by $y$ at the screen:

$$
O^{T} y:=\frac{\partial v^{y}}{\partial \nu} \quad \text { on } \Sigma_{\sigma}^{T}
$$

Notice that the control operator does not change the physical dimension: $\left\langle W^{T} f\right\rangle=\langle f\rangle$, whereas the observation operator changes the dimension as follows: $\left\langle O^{T} y\right\rangle=\langle$ time $\rangle\langle\text { length }\rangle^{-1}\langle y\rangle$.

Let

$$
(y, w)_{\mathrm{int}}:=\int_{\Omega} \frac{\mathrm{d} x}{c^{2}(x)} y(x) w(x)
$$

and

$$
(f, g)_{\mathrm{ext}}:=\int_{\Sigma_{\sigma}^{T}} \mathrm{~d} \Gamma \mathrm{d} t f(\gamma, t) g(\gamma, t)
$$


be the scalar products of functions in $\Omega$ and controls on the screen. We use the subscript "int" in order to emphasize that we are dealing with functions defined into the domain, which is not reachable for an external observer, whereas "ext" indicates functions on the screen, which the observer can operate with. Then $(2.12)$ can be written in the form

$$
\left(W^{T} f, y\right)_{\mathrm{int}}=\left(f, O^{T} y\right)_{\mathrm{ext}}
$$

emphasizing the duality.

A composition of the operators

$$
C^{T}:=O^{T} W^{T}
$$

is an operator mapping controls into observations at the screen. Thus, one can write

$$
C^{T} f=O^{T} W^{T} f=O^{T} u^{f}(\cdot, T) .
$$

The operator $C^{T}$ changes the dimension in the same way as $O^{T}:\left\langle C^{T} f\right\rangle=$ $\langle$ time $\rangle\langle\text { length }\rangle^{-1}\langle f\rangle$.

\subsection{Jumps in the dual system}

Take a smooth function $y$ and introduce the truncated functions

$$
y_{\xi}:= \begin{cases}y & \text { in } \Omega^{\xi} \\ 0 & \text { in } \Omega \backslash \Omega^{\xi}\end{cases}
$$

and

$$
y_{\xi}^{\perp}:=y-y_{\xi}= \begin{cases}0 & \text { in } \Omega^{\xi}, \\ y & \text { in } \Omega \backslash \Omega^{\xi} .\end{cases}
$$

Note that $y_{\xi}^{\perp}$ has a jump at the surface $\Gamma^{\xi}$; in particular, the obvious relation

$$
y_{\xi}^{\perp}(x(\gamma, \xi+0))=y(x(\gamma, \xi))
$$

holds on the part $\sigma^{\xi}$ lighted by the rays in the tube $B^{T}$.

Let us insert $y_{\xi}^{\perp}$ as the Cauchy data in the second condition of (2.10). Such a discontinuous perturbation produces a discontinuous wave carrying a jump at its forward front. The initial jump of the amplitude (2.15) at the 

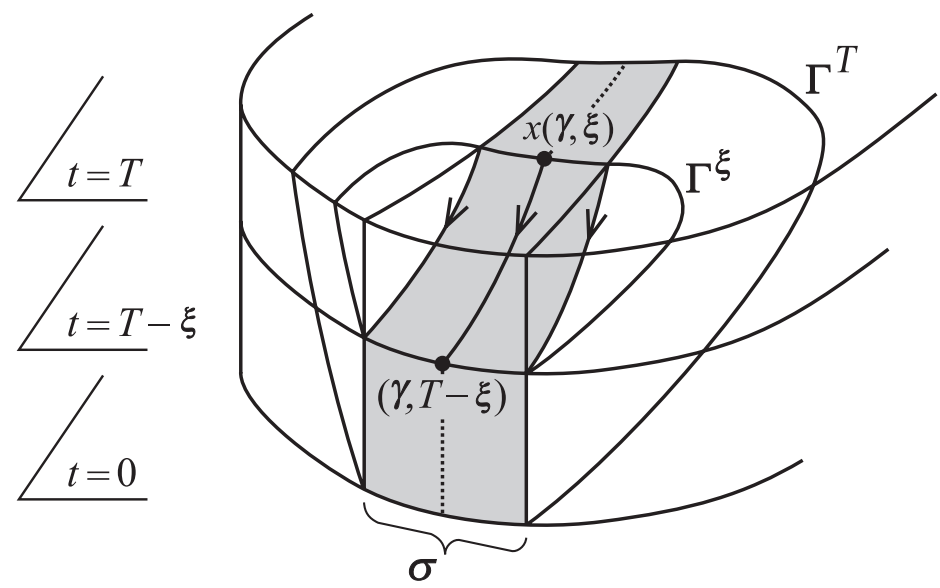

Fig. 3. The jump of $v^{y_{\xi}^{\perp}}$

point $x(\gamma, \xi)$ propagates (in inverted time!) along the ray $r_{\gamma}$ and reaches the boundary at the point $x(\gamma, 0)=\gamma$ at the moment $t=T-\xi$ (see Fig. 3).

Reaching the boundary it produces a jump of the force, whose amplitude can be calculated by the Geometric Optics formula

$$
\frac{\partial v^{y_{\xi}}}{\partial \nu}(\gamma, T-\xi-0)=\sqrt{\frac{J(\gamma, \xi) J(\gamma, 0)}{c(x(\gamma, \xi)) c(x(\gamma, 0))}} y(x(\gamma, \xi)) .
$$

This equality can be derived from (2.8) and the duality relation (2.12) (see, e.g., [3]) or in the framework of the standard ray method [1]. The obvious duality between (2.8) and (2.16) is what physicists call the reciprocity law.

Using the observation operator one can rewrite (2.16) in the form

$$
\left[O^{T} y_{\xi}^{\perp}\right](\gamma, T-\xi-0)=\beta(\gamma, \xi) y(x(\gamma, \xi))
$$

with

$$
\beta(\gamma, \xi):=\sqrt{\frac{J(\gamma, \xi) J(\gamma, 0)}{c(x(\gamma, \xi)) c(x(\gamma, 0))}}
$$

\section{Controllability and wave products}

\subsection{Wave shaping}

Choose a function $y$ (of dimension $\langle y\rangle=\left\langle u^{f}\right\rangle$ ) in the subdomain $\Omega^{T}$ filled with waves and consider the boundary control problem: find a control $f$ 
providing the equality

$$
u^{f}(\cdot, T)=y \quad \text { in } \Omega^{T} .
$$

In other words, the question is whether one can manage a shape of waves from the screen. Being of principal character for our approach, this problem gives the name of the method.

In the general case the answer is the following. For each given $y$ and arbitrary (small) $\varepsilon>0$ one can find a control $f$ such that

$$
\int_{\Omega^{T}} \frac{\mathrm{d} x}{c^{2}(x)}\left|y(x)-u^{f}(x, T)\right|^{2}<\varepsilon .
$$

It means that the set of waves is rich enough to approximate any function (in the mean-square metric). This property of the system (2.2)-(2.4) is called the boundary controllability.

Controllability is a fact of positive character for inverse problems: a very general principle of system theory claims that the richer is the set of states of a dynamical system which an external observer can create by means of controls, the richer is information about the system which the observer can extract from external measurements.

One of the consequences of controllability is the existence of wave basises. A wave basis is a set of waves $u^{f_{1}}(\cdot, T), u^{f_{2}}(\cdot, T), \ldots$ satisfying the orthogonality conditions

$$
\int_{\Omega^{T}} \frac{\mathrm{d} x}{c^{2}(x)} u^{f_{i}}(x, T) u^{f_{j}}(x, T)=\delta_{i j}:= \begin{cases}1, & i=j \\ 0, & i \neq j\end{cases}
$$

such that each $y$ supported in the subdomain $\Omega^{T}$ filled with waves from the screen can be represented in the form of the series

$$
y=\sum_{j=1}^{\infty} c_{j} u^{f_{j}}(\cdot, T)
$$

with the coefficients

$$
c_{j}=\int_{\Omega^{T}} \frac{\mathrm{d} x}{c^{2}(x)} y(x) u^{f_{j}}(x, T) .
$$

We'll return to wave bases later, after preparing some auxiliary results. 


\subsection{Wave products}

Here we show that scalar products of waves can be expressed via measurements at the screen.

Take a control $f$ which acts at the screen. Let

$$
f_{-}(\gamma, t):= \begin{cases}f(\gamma, t), & 0 \leqslant t<T \\ -f(\gamma, 2 T-t), & T \leqslant t \leqslant 2 T\end{cases}
$$

be its odd extension and let

$$
\tilde{f}(\gamma, t):=\int_{0}^{t} f_{-}(\gamma, s) \mathrm{d} s \quad \text { on } \Sigma^{2 T} .
$$

Take $\tilde{f}$ as a control in the problem with the doubled final moment:

$$
\begin{aligned}
& u_{t t}-c^{2}[\Delta u-q u]=0 \quad \text { in } Q^{2 T}, \\
& \left.u\right|_{t=0}=\left.u_{t}\right|_{t=0}=0 \quad \text { in } \Omega, \\
& u=\tilde{f} \quad \text { on } \Sigma^{2 T} ;
\end{aligned}
$$

and let $u=u^{\tilde{f}}(x, t)$ be its solution. We are going to derive the following important relation

$$
\left[O^{T} u^{f}(\cdot, T)\right](\gamma, t)=\frac{1}{2}\left[\frac{\partial u^{\tilde{f}}}{\partial \nu}(\gamma, t)-\frac{\partial u^{\tilde{f}}}{\partial \nu}(\gamma, 2 T-t)\right]
$$

for every point $(\gamma, t)$ of the screen $\Sigma_{\sigma}^{T}$.

Consider the auxiliary problem

$$
\begin{aligned}
& v_{t t}-c^{2}[\Delta v-q v]=0 \quad \text { in } Q^{T}, \\
& \left.v\right|_{t=T}=0,\left.\quad v_{t}\right|_{t=T}=2 u_{t}^{\tilde{f}}(\cdot, T) \quad \text { in } \Omega, \\
& v=0 \quad \text { on } \Sigma^{T} .
\end{aligned}
$$

Since the coefficients of equation (3.18) do not depend on $t$, one has

$$
u_{t}^{\tilde{f}}(\cdot, T)=u^{\tilde{f}_{t}}(\cdot, T)=u^{f_{-}}(\cdot, T)=u^{f}(\cdot, T) ;
$$

hence the derivative in (3.23) can be calculated as follows:

$$
\left.v_{t}\right|_{t=T}=2 u_{t}^{\tilde{f}}(\cdot, T)=2 u^{f}(\cdot, T) .
$$


On the other hand, the solutions of the problems (3.18)-(3.20) and (3.22)-(3.24) are connected by the relation

$$
v(x, t)=u^{\tilde{f}}(x, t)-u^{\tilde{f}}(x, 2 T-t) \quad \text { in } Q^{T}
$$

since the right-hand side of (3.26) satisfies all the conditions (3.22)-(3.24). Differentiating in (3.26) one obtains

$$
\frac{\partial v}{\partial \nu}(\gamma, t)=\frac{\partial u^{\tilde{f}}}{\partial \nu}(\gamma, t)-\frac{\partial u^{\tilde{f}}}{\partial \nu}(\gamma, 2 T-t) \quad \text { on } \Sigma_{\sigma}^{T}
$$

Recalling the definition of the observation operator, with regard to (3.25) we get

$$
\left\{O^{T}\left[2 u^{f}(\cdot, T)\right]\right\}(\gamma, t)=\frac{\partial v}{\partial \nu}(\gamma, t) \stackrel{(3.27)}{=} \frac{\partial u^{\tilde{f}}}{\partial \nu}(\gamma, t)-\frac{\partial u^{\tilde{f}}}{\partial \nu}(\gamma, 2 T-t),
$$

which leads to (3.21).

In accordance with (2.14) the last relation can be written in the form of representation

$$
\left(C^{T} f\right)(\gamma, t)=\frac{1}{2}\left[\frac{\partial u^{\tilde{f}}}{\partial \nu}(\gamma, t)-\frac{\partial u^{\tilde{f}}}{\partial \nu}(\gamma, 2 T-t)\right]
$$

on the screen.

Choose a pair of controls $f, g$; by virtue of (3.28) one has

$$
\begin{aligned}
& \left(u^{f}(\cdot, T), u^{g}(\cdot, T)\right)_{\mathrm{int}} \stackrel{(2.13)}{=}\left(O^{T} u^{f}(\cdot, T), g\right)_{\mathrm{ext}} \stackrel{(2.14)}{=}\left(C^{T} f, g\right)_{\mathrm{ext}} \\
& \stackrel{(3.28)}{=} \frac{1}{2} \int_{\Sigma_{\sigma}^{T}} \mathrm{~d} \Gamma \mathrm{d} t\left[\frac{\partial u^{\tilde{f}}}{\partial \nu}(\gamma, t)-\frac{\partial u^{\tilde{f}}}{\partial \nu}(\gamma, 2 T-t)\right] g(\gamma, t) .
\end{aligned}
$$

These relations are interesting and important. An external observer operating at the boundary can set controls and create waves but cannot see the waves in themselves. Nevertheless, (3.29) gives him the possibility to calculate the scalar products of these invisible waves through the data $\partial u^{\tilde{f}} / \partial \nu$ and $g$ on the screen $\Sigma_{\sigma}^{T}$, which are at his disposal. A.S.Blagovestchenskii was the first who payed attention to this remarkable fact. 


\subsection{Wave basis}

The wave bases introduced at the end of Section 3.1 provide the main device of the procedure, which makes the waves visible through the boundary measurements.

Fix $\xi$ provided $0<\xi<T$ and choose a complete system of controls $g_{1}, g_{2}, \ldots$ supported on the part $\sigma \times[T-\xi, T]$ of the screen (so that each $g_{j}$ is switched on with delay $T-\xi$ and acts $\xi$ units of time). A completeness means that controls supported on the same part $\sigma \times[T-\xi, T]$ can be expanded as $f=\sum_{j=1}^{\infty} \alpha_{j} g_{j}$. Notice that in accordance with (2.5), the corresponding waves $u^{g_{j}}(\cdot, T)$ are supported in the subdomain $\Omega^{\xi}$.

The external observer, which possesses the controls $g_{j}$ and the measurements $\partial u^{\tilde{g}_{j}} / \partial \nu$, can modify the system $g_{1}, g_{2}, \ldots$ by the Schmidt process as follows:

$$
\begin{aligned}
& g_{1}^{\prime}:=g_{1}, \quad f_{1}:=\left(C^{T} g_{1}^{\prime}, g_{1}^{\prime}\right)_{\mathrm{ext}}^{-1 / 2} g_{1}^{\prime} ; \\
& g_{2}^{\prime}:=g_{2}-\left(C^{T} g_{2}, f_{1}\right)_{\mathrm{ext}} f_{1}, \quad f_{2}:=\left(C^{T} g_{2}^{\prime}, g_{2}^{\prime}\right)_{\mathrm{ext}}^{-1 / 2} g_{2}^{\prime} \text {; } \\
& g_{j}^{\prime}:=g_{j}-\sum_{k=1}^{j-1}\left(C^{T} g_{j}, f_{k}\right)_{\mathrm{ext}} f_{k}, \quad f_{j}:=\left(C^{T} g_{j}^{\prime}, g_{j}^{\prime}\right)_{\mathrm{ext}}^{-1 / 2} g_{j}^{\prime} ; \\
& \ldots \ldots \ldots \ldots \ldots \ldots \ldots \ldots \ldots \ldots \ldots \ldots
\end{aligned}
$$

calculating all the "ext"-products by (3.28) and (3.29). The obtained system $f_{1}, f_{2}, \ldots$ is complete on $\sigma \times[T-\xi, T]$ and satisfies

$$
\left(C^{T} f_{i}, f_{j}\right)_{\mathrm{ext}}=\delta_{i j}
$$

By virtue of (3.29) the corresponding waves $u^{f_{1}}(\cdot, T), u^{f_{2}}(\cdot, T), \ldots$ satisfy

$$
\left(u^{f_{i}}(\cdot, T), u^{f_{j}}(\cdot, T)\right)_{\mathrm{int}}=\left(C^{T} f_{i}, f_{j}\right)_{\mathrm{ext}}=\delta_{i j},
$$

i.e., constitute an orthogonal normalized system in $\Omega^{\xi}$. A deep fact is that, due to controllability, this system turns out to be a basis in $\Omega^{\xi}$.

Thus, not seeing the waves in themselves, the external observer, nevertheless, is able to construct a system of controls $f_{1}, f_{2}, \ldots$ producing the wave basis $u^{f_{1}}(\cdot, T), u^{f_{2}}(\cdot, T), \ldots$ in the prescribed subdomain $\Omega^{\xi}$ reachable for waves generated at the screen. 
As a computational problem the orthogonalization by (3.30) is equivalent to inversion of the ill-posed Gram matrix $G_{i j}:=\left(C^{T} g_{i}, g_{j}\right)_{\text {ext }}$ of large size (see [4]).

The wave basis can be used in the truncation procedure: if $y$ is a function on $\Omega$, then its cut-off function $y_{\xi}$ can be represented as follows:

$$
y_{\xi}=\sum_{j=1}^{\infty}\left(y, u^{f_{j}}(\cdot, T)\right)_{\mathrm{int}} u^{f_{j}}(\cdot, T) .
$$

In the important particular case of $y=u^{f}(\cdot, T)$ one has

$$
u^{f}(\cdot, T)_{\xi}=\sum_{j=1}^{\infty}\left(u^{f}(\cdot, T), u^{f_{j}}(\cdot, T)\right)_{\mathrm{int}} u^{f_{j}}(\cdot, T) \stackrel{(3.29)}{=} \sum_{j=1}^{\infty}\left(C^{T} f, f_{j}\right)_{\mathrm{ext}} u^{f_{j}}(\cdot, T),
$$

so that the external observer can find the coefficients of this expansion by (3.29).

\section{Visualization of waves}

\subsection{Portraits}

Let $y$ be a function in $\Omega$. A function on $\Sigma_{\sigma}^{T}$

$$
\tilde{y}(\gamma, t):=\beta(\gamma, t) y(x(\gamma, t)),
$$

( $\beta$ is defined in (2.17)) is called a portrait (or image) of $y$ on the screen.

Thus, up to the factor $\beta$, the portrait is just the result of point-wise transferring a function from the tube to the screen. This is the basic notion of our approach. Note that since $\tilde{y}$ is determined by the values $\left.y\right|_{B^{T}}$, it would be more precise to speak about the portrait of the part of $y$ in $B^{T}$.

In the rest of the paper, we show that the external observer, which possesses a complete system of controls $g_{1}, g_{2}, \ldots$ on $\Sigma_{\sigma}^{T}$ and the corresponding measurements $\partial u^{\tilde{g}_{1}} / \partial \nu, \partial u^{\tilde{g}_{2}} / \partial \nu, \ldots$ on $\Sigma_{\sigma}^{2 T}$, is able to reconstruct the portrait of any wave $u^{f}(\cdot, T)$, i.e., can make the wave to be visible. 


\subsection{Amplitude formula}

The procedure for recovering the portraits is based on the Geometric Optics formulas. Combining the definition (4.32) with (2.17) one gets the equality

$$
\tilde{y}(\gamma, \xi)=\left[O^{T} y_{\xi}^{\perp}\right](\gamma, T-\xi-0), \quad(\gamma, \xi) \in \Sigma_{\sigma}^{T},
$$

which is called the amplitude formula: it represents the portrait as a collection of amplitudes of the jumps, which are induced by the truncation of $y$, pass through the medium, and are detected at the screen.

Now we are going to insert $y=u^{f}(\cdot, T)$ in (4.33). We begin with a representation of the truncated wave. Applying the observation operator to (3.31) we obtain

$$
O^{T} u^{f}(\cdot, T)_{\xi}=\sum_{j=1}^{\infty}\left(C^{T} f, f_{j}\right)_{\mathrm{ext}} O^{T} u^{f_{j}}(\cdot, T) \stackrel{(2.14)}{=} \sum_{j=1}^{\infty}\left(C^{T} f, f_{j}\right)_{\mathrm{ext}} C^{T} f_{j},
$$

which yields

$$
O^{T} u^{f}(\cdot, T)_{\xi}^{\perp}=O^{T}\left[u^{f}(\cdot, T)-u^{f}(\cdot, T)_{\xi}\right]=C^{T} f-\sum_{j=1}^{\infty}\left(C^{T} f, f_{j}\right)_{\mathrm{ext}} C^{T} f_{j} .
$$

Substituting this in (4.33) we arrive at the final formula

$$
\left[u^{\widetilde{f}(\cdot, T)}\right](\gamma, \xi)=\left\{C^{T} f-\sum_{j=1}^{\infty}\left(C^{T} f, f_{j}\right)_{\mathrm{ext}} C^{T} f_{j}\right\}(\gamma, T-\xi-0),
$$

which is a main device for visualization. It represents the "slice" of the portrait corresponding to a fixed $\xi$ in terms of the boundary measurements.

\subsection{Visualizing the portraits of waves}

Let us summarize our results. If the external observer can measure the values $\partial u^{\tilde{g}} / \partial \nu$ on the screen $\Sigma_{\sigma}^{2 T}$ for rich enough reserve of controls $g$, he is able to recover a portrait of any wave by the following procedure.

Step 1 (orthogonalization of controls). Fix $\xi: 0<\xi<T$ and choose a complete system $g_{1}, g_{2}, \ldots$ of controls supported on the part $\sigma \times[T-\xi, T]$ of the screen $\Sigma_{\sigma}^{T}$. Construct the system $f_{1}, f_{2}, \ldots$ (see (3.30)) by calculating the "ext"-products by (3.29). 
Step 2 (reconstruction of "slice"). Find $C^{T} f_{1}, C^{T} f_{2}, \ldots$ with the help of (3.28). Specifying $f$, recover $\left[u^{f(\cdot, T)}\right](\gamma, \xi)$ for all $\gamma \in \sigma$ by means of (4.34).

Step 3 (reconstruction of the portrait). Varying $\xi$ and repeating steps 1 and 2 recover $u^{f(\cdot, T)}$ on the screen.

\subsection{Recovering the potential}

Assume that the wave speed is constant: $c=1$, whereas the (unknown) potential $q$ is variable. In this case the c-metric is Euclidean, the rays $r_{\gamma}$ are straight lines, and the factor $\beta$ entering in the definition of portraits can be regarded as known. Moreover, we know the relation between the ray and Cartesian coordinates. Therefore, we can recover the portrait $\widetilde{u^{f(\cdot, T)} \text { and }}$ then find the wave itself at each point $x(\gamma, \xi)$ in the tube $B^{T}$ by the rule

$$
u^{f}(x(\gamma, \xi), T)=\beta^{-1}(\gamma, \xi)\left[\widetilde{\left.u^{f(\cdot, T}\right)}\right](\gamma, \xi) .
$$

Finally, specifying $f$ and recovering the waves $u^{f}(\cdot, T)$ and $u_{t t}^{f}(\cdot, T)=u^{f_{t t}}(\cdot, T)$ through their portraits by $(4.35)$, one can find the potential in the tube $B^{T}$ from the wave equation

$$
q(x)=\left[u^{f}(x, T)\right]^{-1}\left\{\Delta u^{f}(x, T)-u^{f_{t t}}(x, T)\right\} .
$$

Another way is to take the control $f(\gamma, t)=\theta(t-(T-\tau))$, visualize the corresponding wave $u^{f}(\cdot, T)$ in the tube $B^{T}$, and extract the potential from the jump of the wave and its derivatives at the forward front $\sigma^{\tau}$ by means of the well-known Geometric Optics formulas.

If the potential $q=0$ and the speed $c$ is variable and unknown, we meet another situation: the c-metric is not Euclidean and the rays $r_{\gamma}$ and factor $\beta$ are unknown. The correspondence $(\gamma, \xi) \leftrightarrow x(\gamma, \xi)$ between coordinates, which was used for recovering the potential is also unknown. So, this case requires some additional work.

\subsection{Portraits of harmonic functions}

Let $a=a(x)$ be a harmonic function: $\Delta a(x)=0$ in $\Omega$. In the calculations following below, we use the representation

$$
u^{f}(x, T)=\int_{0}^{T} \mathrm{~d} t(T-t) u_{t t}^{f}(x, t),
$$


which holds due to zero Cauchy data (2.3). Also, we assume that the control $f$ is smooth and vanishes near $t=0$ so that the wave $u^{f}(x, T)$ is supported in $\Omega^{T}$ and vanishes near its forward front $\Gamma^{T}$. Owing to the latter the surface integrals over the front vanish and we have the equalities

$$
\begin{gathered}
\left(a, u^{f}(\cdot, T)\right)_{\mathrm{int}}=\int_{\Omega} \frac{\mathrm{d} x}{c^{2}(x)} a(x) u^{f}(x, T) \\
\stackrel{(4.36)}{=} \int_{\Omega} \frac{\mathrm{d} x}{c^{2}(x)} a(x) \int_{0}^{T} \mathrm{~d} t(T-t) u_{t t}^{f}(x, t) \\
=\int_{0}^{T} \mathrm{~d} t(T-t) \int_{\Omega} \mathrm{d} x \frac{a(x) u_{t t}^{f}(x, t)}{c^{2}(x)} \\
\stackrel{(2.2)}{\stackrel{\text { with }}{=}} q=0 \int_{0}^{T} \mathrm{~d} t(T-t) \int_{\Omega} \mathrm{d} x a(x) \Delta u^{f}(x, t) \\
=\int_{0}^{T} \mathrm{~d} t(T-t) \int_{\Gamma} \mathrm{d} \Gamma\left[a(\gamma) \frac{\partial u^{f}}{\partial \nu}(\gamma, t)-\frac{\partial a}{\partial \nu}(\gamma) f(\gamma, t)\right] .
\end{gathered}
$$

Thus, using (4.37), the external observer can find the product of any harmonic function and invisible wave through the boundary measurements. Note that, in this case, the observer needs to know $\partial u^{f} / \partial \nu$ not only on $\sigma$ but on a wider part of $\Gamma$ filled with waves at the moment $t=T^{1}$. Equality (4.37) enables one to describe the expansion of the truncated harmonic function in the wave basis:

$$
\begin{gathered}
a_{\xi}=\sum_{j=1}^{\infty} \alpha_{j} u^{f_{j}}(\cdot, T), \\
\alpha_{j}=\left(a, u^{f_{j}}(\cdot, T)\right)_{\text {int }}=\int_{\Sigma^{T}} \mathrm{~d} \Gamma \mathrm{d} t(T-t)\left[a(\gamma) \frac{\partial u^{f_{j}}}{\partial \nu}(\gamma, t)-\frac{\partial a}{\partial \nu}(\gamma) f_{j}(\gamma, t)\right]
\end{gathered}
$$

and then find

$$
O^{T} a_{\xi}=\sum_{j=1}^{\infty} \alpha_{j} O^{T} u^{f_{j}}(\cdot, T) \stackrel{(2.14)}{=} \sum_{j=1}^{\infty} \alpha_{j} C^{T} f_{j} .
$$

The latter enables one to visualize the portrait:

$$
\tilde{a}(\gamma, \xi)=\left\{O^{T}\left[a_{T}-a_{\xi}\right]\right\}(\gamma, T-\xi-0) .
$$

\footnotetext{
${ }^{1}$ however, see Comments at the end of the paper.
} 
So, the external observer can reconstruct the portrait of any harmonic function by the scheme:

(i) for each $\xi: 0<\xi \leqslant T$, prepare the system $f_{1}, f_{2}, \ldots$ on $\sigma \times[T-\xi, T]$ and then find $C^{T} f_{1}, C^{T} f_{2}, \ldots$;

(ii) get the coefficients $\alpha_{1}, \alpha_{2}, \ldots$ by (4.38) and find $O^{T} a_{\xi}$ by (4.39);

(iii) reconstruct the portrait by (4.40).

Now we are ready to recover the speed in the tube $B^{T}$.

\subsection{Recovering the speed}

Introduce a function $1(x)=1$ in $\Omega$ and the Cartesian coordinate functions $\pi_{1}(x), \pi_{2}(x), \pi_{3}(x): \pi_{i}(x)=x^{i}$ for $x=\left(x^{1}, x^{2}, x^{3}\right)$. All of these functions are harmonic; therefore, we can determine their portraits

$$
\tilde{1}(\gamma, t)=\beta(\gamma, t), \quad \tilde{\pi}_{i}(\gamma, t)=\beta(\gamma, t) \pi_{i}(x(\gamma, t))
$$

and then find

$$
x^{i}(\gamma, t)=\pi_{i}(x(\gamma, t))=\frac{\tilde{\pi}_{i}(\gamma, t)}{\tilde{1}(\gamma, t)}, \quad i=1,2,3 .
$$

So, for each point $(\gamma, t)$ of the screen, we find the point $x(\gamma, t)$ in the tube. In other words, fixing $\gamma$ and varying $t$ the external observer can see how the point $x(\gamma, t)$ moves along the ray $r_{\gamma}$ in the interior of $\Omega$. Then, at last, the observer can find the speed in $B^{T}$ by the obvious equality

$$
c(x(\gamma, t))=\left\{\sum_{i=1}^{3}\left[\frac{\mathrm{d}}{\mathrm{d} t} x^{i}(\gamma, t)\right]^{2}\right\}^{1 / 2} .
$$

\section{Comments}

- There are the versions of the BC-method, which recover $\left.c\right|_{B^{T}}$ via $R^{2 T}$ given on $\sigma$ only (i.e., without the use of measurements outside $\sigma$ ). The first version [2] is a kind of the sample algorithm. A more promoted variant is proposed in [6], section 4.1. 
- As is shown in $[4,5,6]$, the BC-method can be used as a background of numerical algorithms. Successful results on numerical testing were obtained by V.Yu.Gotlib. To our great sorrow, this work had to be suspended because of his death. Later on, this activity was renewed in [6]; the most promoted results see in [7].

- The problem with the Neumann boundary controls $\partial u / \partial \nu=f$ on $\Sigma^{T}$ may be treated along the same lines. In this case, the amplitude formula contains one additional differentiation and looks like that:

$$
\tilde{y}(\gamma, \xi)=\left[\frac{\partial}{\partial t} O^{T} y_{\xi}^{\perp}\right](\gamma, T-\xi-0) .
$$

\section{References}

1. V. M. Babich and V. S. Buldyrev. Short-Wavelength Diffraction Theory. Asymptotic Methods. Springer-Verlag, Berlin, 1991.

2. M. I. Belishev. Boundary control in reconstruction of manifolds and metrics (the BC-method). Inverse Problems (1997) 13, No. 5, R1-R45.

3. M. I. Belishev and A. S. Blagovestchenskii. Dynamical Inverse Problems of the Wave Propagation Theory. St.-Petersburg State University, St.-Petersburg, 1999 (in Russian).

4. M. I. Belishev and V. Yu. Gotlib. Dynamical variant of the BC-method: theory and numerical testing. J. Inverse Ill-Posed Problems (1999) 7, No. 3, 221-240.

5. M. I. Belishev, V. Yu. Gotlib, and S. A. Ivanov. The BC-method in multidimensional spectral inverse problems: theory and numerical illustrations. ESAIM Control Optim. Calc. Var. (1997) 2, 307-327.

6. M.I.Belishev, I.B.Ivanov, I.V.Kubyshkin, and V.S.Semenov. Numerical testing in determination of sound speed from a part of boundary by the bc-method. J. Inverse Ill-Posed Problems (2016) 23, No. 5, 221-240.

7. I.B.Ivanov, M.I.Belishev, V.S.Semenov. The reconstruction of sound speed in the Marmousi model by the boundary control method. http://arxiv.org/abs/1609.07586. 